\title{
Shielding Calculations on Waste Packages - The Limits and Possibilities of different Calculation Methods by the example of homogeneous and inhomogeneous Waste Packages
}

\author{
Mike Adams and Silva Smalian ${ }^{a}$ \\ TÜV NORD EnSys Hannover GmbH \& Co. KG, Hannover, Germany
}

\begin{abstract}
For nuclear waste packages the expected dose rates and nuclide inventory are beforehand calculated. Depending on the package of the nuclear waste deterministic programs like MicroShield ${ }^{\circledR}$ provide a range of results for each type of packaging. Stochastic programs like "Monte-Carlo N-Particle Transport Code System" (MCNPß) on the other hand provide reliable results for complex geometries. However this type of program requires a fully trained operator and calculations are time consuming. The problem here is to choose an appropriate program for a specific geometry. Therefore we compared the results of deterministic programs like MicroShield $®$ and stochastic programs like MCNP®. These comparisons enable us to make a statement about the applicability of the various programs for chosen types of containers. As a conclusion we found that for thin-walled geometries deterministic programs like MicroShield ${ }^{\circledR}$ are well suited to calculate the dose rate. For cylindrical containers with inner shielding however, deterministic programs hit their limits. Furthermore we investigate the effect of an inhomogeneous material and activity distribution on the results. The calculations are still ongoing. Results will be presented in the final abstract.
\end{abstract}

\section{Introduction}

In medicine, in nuclear plants or in research accumulating radioactive waste has to be disposed of properly. In Germany the radioactive waste which cannot be reused or released has to temporarily be stored and finally be disposed of in a Federal final disposal site [1]. Before final disposal these wastes have to be submitted to a qualified conditioning process, during which the protection of staff members and the population from radioactive contamination is of highest priority.

To protect the population the dose rates and the nuclide inventory of radioactive waste ready for interim or final storage are strictly limited by both, the acceptance criteria of interim and final disposal sites [2] and by the transportation law. This has to be taken into consideration when conceiving a loading concept for radioactive waste campaigns and sizing radioactive waste containers.

To ensure these limitations the expected dose rates and nuclide inventory are beforehand calculated with suitable software. The user has to choose the right program for each type of packaging. Depending on the calculation parameters chosen deterministic programs with an easily accessible user interface such as MicroShield ${ }^{\circledR}$ already provide a range of results for simple geometries. These results, however, may differ by

\footnotetext{
${ }^{\mathrm{a}}$ Corresponding author: ssmalian@ $@$ tuev-nord.de
}

factor 4 from each other and therefore can't be used efficiently for loading concepts and container design. Stochastic programs using the "Monte-Carlo N-Particle Transport Code System" (MCNP $®)$ on the other hand provide reliable results for complex geometries, but require a fully trained operator. The question therefore is: Which program is the most efficient for which geometry? And how does an inhomogeneous material and activity distribution affect the results?

Comparing the results calculated with both, MicroShield $\AA$ and $M C N P \AA$, we have examined the problem of the input-parameters with MicroShield $\AA$ for some containers relevant for temporary and final disposal of waste. In addition, we limited the range of application of MicroShield $\AA$ for these containers.

Our comparisons enable us to make a statement about the applicability of the various programs for chosen types of containers and distributions.

\section{Calculation Method}

To check the observance of the waste acceptance criteria for the dose rates and the nuclide inventory, conclusions must be drawn from dose rate measurements at containers to the activity inventory or, when conceiving loading concepts, from the known activity to 
the dose rate expected at the containers. For this purpose several shielding-programs are available of which we compared two.

\subsection{MicroShield}

MicroShield $\AA$, a product of Grove Software, Inc. was developed in the early 1990s in Virginia, USA, to perform shielding calculation. It is limited to and internationally recognized for a selection of/number of standard geometries. For multi-layer shields it can only be used under reservation and - as will be shown - with deeper understanding. The program is continuously being further developed and is now available in version 10 .

\subsection{MCNP}

The program MCNP $\AA$ is a trademark of Los Alamos National Security, Los Alamos National Laboratory and simulates the transport of particles through matter by using Monte Carlo algorithms. It thus allows the analysis of complex three-dimensional geometries with almost any material and source distribution (see fig. 12). Modelling effort and computing time are the only limiting factors. The program is constantly being further developed and is now available in version 6.1.

During the simulation of particle transport problems by means of the Monte Carlo method, each particle from its generation until its disappearance (absorption or leaving the modelled area) - is monitored/examined. By using probability density functions and generated random numbers events for the particles are generated. The probability density functions are available for each material stored in tables.

The user provides all the relevant data as an input file, the drawing up of which is a complex task with MCNP $\AA$, therefore more error-prone than MicroShield and requiring a trained operator.

\section{Comparison of the results}

We have modeled different types of containers in constant conditions with both programs and compared the results. Our goal is to raise the awareness of the problem and make a statement about the range of application of the two differently convenient calculation methods, "MicroShield" and "MCNP", in regard to the reliability of the results provided by them.

In the considered volume sources, a homogeneous and an inhomogeneous distribution of the waste product and activity were assumed.

\subsection{Thin walled geometries with homogeneous material and activity distribution}

The MCNP® results are well in agreement with the MicroShield results for the build-up factor of the source. The MicroShield results differ by factor $\sim 1,1$ from each other. The build-up factor of the source clearly dominates these geometries, so that MicroShield $\AA$ - provided the build-up factor is properly chosen - can be used to calculate the dose rate. Further marginal conditions, which are not modeled (e.g. inhomogeneous density or source distribution), lead to larger deviations than the choice of the calculation procedure.

\subsection{Thin walled geometries with inhomogeneous material and activity distribution}

The calculations are still ongoing. Results will be presented in the final abstract.

\subsection{Thick walled geometries with homogeneous material and activity distribution}

The results for a cylindrical container filled with iron (height $150 \mathrm{~cm}$, diameter $106 \mathrm{~cm}$, wall thickness $16 \mathrm{~cm}$, top and bottom $18 \mathrm{~cm}$, density $7 \mathrm{~g} / \mathrm{cm}^{3}$ ) and with $100 \mathrm{~mm}$ lead shielding inside are depending on the waste density modeled. The dose rate is calculated at a one-meter distance from the waste package at half height. The MicroShield ${ }^{\circledR}$ results for different build-up factors in otherwise constant conditions differ by factor 4 . The MCNP ${ }^{\circledR}$ results are within this range and are either under-estimated by factor 0.5 or over-estimated by factor 2 by the MicroShield $\AA$ results.

\subsection{Thick walled geometries with inhomogeneous material and activity distribution}

The calculations are still ongoing. Results will be presented in the final abstract.

\section{Conclusion}

Our comparisons make it possible to judge about the applicability of the various programs for chosen types of waste packages with homogenous material and activity distribution. For the thin-walled geometries the program MicroShield ${ }^{\circledR}$ is well suited to calculate the dose rate. For this purpose the source's dominating build-up factor, which leads to the below mentioned deviations from MCNP ${ }^{\circledR}$, has to be chosen. For some thick walled geometries, however, the program hits its limits. These examples clearly show the problem of choosing the right build-up factor at MicroShield $\AA$.

We expect to make a statement about the applicability of the various programs for chosen types of waste packages with inhomogeneous material and activity distribution. The calculations are still ongoing.

\section{References}

\section{StrlSchV}

Verordnung über den Schutz vor Schäden durch ionisierende Strahlen (StrahlenschutzverordnungStrlSchV) vom 20. Juli

2. BfS

Anforderungen an endzulagernde radioaktive Abfälle 
(Endlagerungsbedingungen) Stand: Dezember 1995 -

Schachtanlage Konrad-, ET-IB-79 